\title{
Scrotal enlargement and constipation in a male rat
}

Genaro A. Coria-Avila, DVM, MSc, PhD ${ }^{1,2}$, Jorge Manzo, PhD², Pablo Pacheco, MD, PhD 2,3 \& James G. Pfaus, PhD ${ }^{1}$

We purchased a batch of 40 adult LongEvans male rats (300-350 g body weight) from a laboratory animal supplier for studies on sexual behavior. We kept the males in pairs in shoebox cages in a colony room maintained at approximately $22 \pm 4{ }^{\circ} \mathrm{C}$ on a reverse 12:12-h light/dark cycle with free access to commercial laboratory rat chow and water. All the males received sexual conditioning, which consisted of 30-minute copulatory trials every 4 days with ovariectomized, hormone-primed females.

During an extended holiday, the male rats were not in use for two weeks. Because of the absence of researchers during this period, the rats were not handled or observed as frequently as they had been prior to the holiday. Over the holiday, the animal caretaker reported that one rat had a slight enlargement of the scrotum. Physical examination revealed a scrotum with a circumference of $10 \mathrm{~cm}$ (other males of similar weight

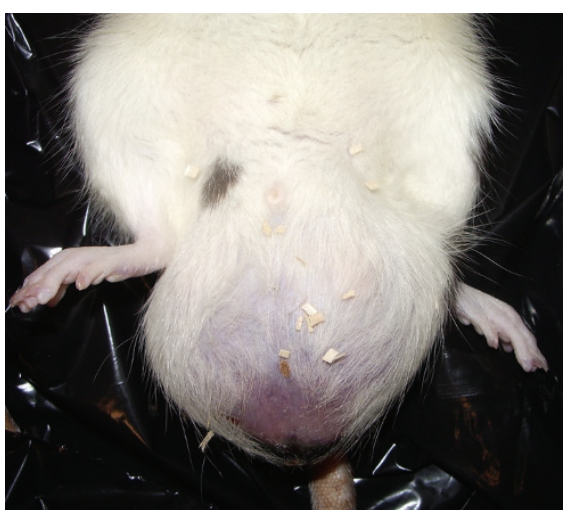

FIGURE 1 | A Long-Evans male with severe scrotal distention. The scrotum felt cold when palpated and the rat did not react to the scrotal examination. had scrotal circumferences of 7.5-8 cm) that was slightly cold to the touch. The rat showed no signs of pain in its cage or upon examination. It had normal locomotion, normal body temperature $\left(37^{\circ} \mathrm{C}\right)$, and seemed bright and alert. Given that the males were housed two rats per cage, it was not possible to assess food intake, defecation, or other parameters for this animal. We decided to continue observations.

Three days later, the animal caretaker reported that the same rat now had a severe scrotal enlargement (Fig. 1). By this time, the scrotal circumference had increased to $\sim 20 \mathrm{~cm}$. Physical examination, however, still revealed normal body temperature and normal gait, reflexes, and proprioception. The rat was alert and there were no signs of dehydration or starvation, although it had clinical signs of dyspnea. The scrotum was unusually cold to the touch and its contents turgid. Abdominal palpation revealed a colon greatly distended by fecal pellets, which we interpreted as constipation. We decided to euthanize the rat with $\mathrm{CO}_{2}$ and perform necropsy.

Observation of the thoracic cavity revealed hyperemic lungs. In the abdominal cavity, we observed dilated vessels and a cecum and colon distended with feces. The prostate was hyperemic and enlarged (Fig. 2). Dissection of the scrotum revealed normal testicles, with no apparent signs of inflammation or abnormal content. The vas deferens, the bladder, and the penis looked normal. On closer inspection, we saw that the scrotal enlargement was due to an adjacent sac, located between the tunica vaginalis, the rectum, and the scrotal skin (Fig. 2). Dissection of this sac revealed an abnormal

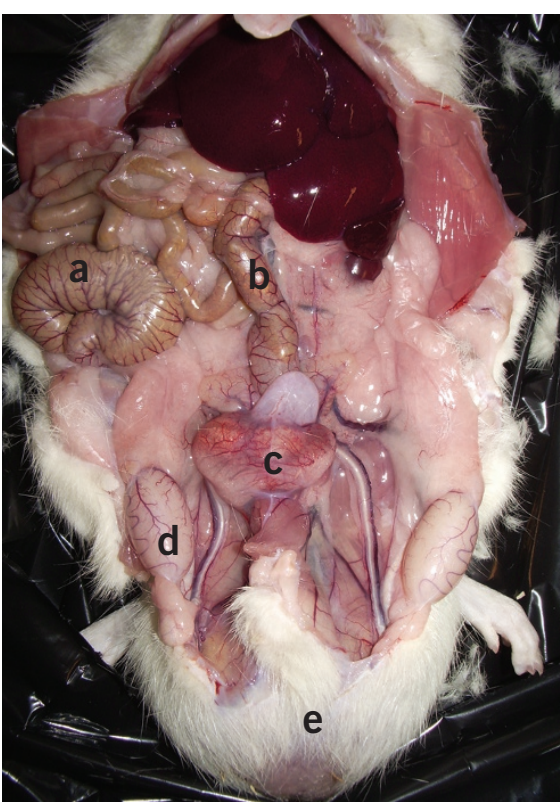

FIGURE 2 | Ventral view of the abdominal cavity of the rat in Figure 1. (a) Cecum (b) rectum (c) prostate (d) right testicle and (e) abnormal scrotal sac. The colon, which is covered by the jejunum in this image, is distended with fecal matter. The enlarged scrotum resulted from the feces-filled cavity.

cavity filled with $\sim 50 \mathrm{~g}$ of feces. The wall of the cavity had signs of necrosis and there was a foul odor. Further dissection demonstrated that the rectum and the anus were no longer connected; we observed a loss of mucosal continuity. Based on the clinical signs and necropsy, what do you think was the problem? How frequent is it?

\section{What's your diagnosis?}

\footnotetext{
${ }^{1}$ Center for Studies in Behavioral Neurobiology, Department of Psychology, Concordia University, 7141 Sherbrooke West, Montréal, QC H4B 1R6, Canada. ${ }^{2}$ Instituto de Neuroetolgía, Universidad Veracruzana, Av. Luis Castelazo s/n, Col. Industrial Las Animas, Xalapa, Veracruz C.P. 91190 , México. ${ }^{3}$ Departmento de Biología Celular, Instituto de Investigaciones Biomédicas, Unidad Periférica Xalapa, Universidad Nacional Autónoma de México. Correspondence should be addressed to G.A.C.-A. (coria75@yahoo.com).
} 